\title{
RELACIONES ENTRE LOS POBLADORES RURALES Y LOS CARNÍVOROS ALTOANDINOS DEL DISTRITO DE ANCO, CENTRO - SUR DEL PERÚ
}

\section{RELATIONSHIPS BETWEEN RURAL SETTLERS AND HIGHLAND CARNIVORES IN THE DISTRICT OF ANCO, CENTER - SOUTH OF PERU}

\author{
Iris Deustua Aris ${ }^{1}$, Marta Williams León de Castro ${ }^{2}$ y Pedro Vásquez Ruesta ${ }^{3}$
}

\begin{abstract}
Resumen
Se realizaron 75 entrevistas semi - estructuradas a los pobladores rurales del distrito de Anco en Agosto de 2006 con el fin de describir las relaciones entre el ser humano y los carnívoros altoandinos. El 100\% de los entrevistados afirmó que existe un gran conflicto en la convivencia con los carnívoros altoandinos. Este se debe a la incidencia de pérdidas económicas producidas por la depredación de animales domésticos y/o pérdidas en la producción de los cultivos agrícolas. El 52\% de los pobladores reconoció haber cazado algún carnívoro altoandino debido a este conflicto. El 44\% tiene una actitud negativa hacia estas especies por su comportamiento depredador, mientras que el 56\% posee una actitud positiva basada en percepciones de atracción y apreciación por su inteligencia, fuerza, poder, valentía y belleza. Los carnívoros altoandinos son usados con fines medicinales a través de la grasa, alimenticios a través de la carne y culturales o artesanales a través de la piel, aunque la intensidad de su uso y valoración ha disminuido a través de los años.
\end{abstract}

Palabras clave: pobladores rurales, carnívoros altoandinos, relaciones hombre-fauna silvestre, usos tradicionales

\begin{abstract}
Semi - structured interviews were conducted on 75 rural settlers of the district of Anco during August 2006 aimed at describing relationships between rural settlers and highland carnivores. One hundred percent of those interviewed stated that there is a huge conflict with highland carnivores. The conflict can be traced to economic losses produced by highland carnivores due to predation of domestic animals and lost crops. Because of the conflict, rural settlers (52\%) recognized having hunted highland carnivores. A percentage of $44 \%$ of the rural settlers have a negative attitude towards these species due to their predator behavior, whereas $56 \%$ have a positive attitude based on attraction and appreciation of their intelligence, force, courage and beauty. Highland carnivores are used for the following purposes: fat is used in medicine, meat is used as food and skin is used culturally or for craftsmanship purposes, although the intensity of their use and value has diminished through the years.
\end{abstract}

Key words: rural settlers, highland carnivores, wildlife and human relationships, wildlife use

\section{Introducción}

En nuestro país son muy pocos los trabajos que describen las relaciones entre los pobladores rurales y la fauna silvestre; de igual forma, son escasos los estudios referidos en particular a los carnívoros altoandinos.

La historia de la interacción entre seres humanos y carnívoros silvestres siempre ha estado plagada de conflictos. Los carnívoros han sido perseguidos por siglos, debido a la depredación de animales domésticos, por tomar vidas humanas o simplemente por temor (Sillero, 2000). Del mismo modo, han sido cazados como consecuencia de arraigadas tradiciones rurales que los perciben como una amenaza constante por ser considerados competidores del hombre (Mishra, 1997), debido a que sus presas más importantes, son también consumidas por los pobladores rurales (Ojasti, 1984; Hoogesteijn, 1993). Igualmente, es posible que exista una necesidad instintiva del hombre para darles caza sin ningún motivo en particular (Kruuk, 1976).

Se ha reportado la depredación de animales domésticos en veinticuatro especies de carnívoros silvestres a nivel mundial (The UICN / SSC Canid Specialist Group, 1990). La principal causa de mortandad en los carnívoros es consecuencia de este conflicto con el ser humano (Woodroofe \& Ginsberg, 1998). Debido a que este conflicto es temporalmente resuelto con la eliminación del carnívoro responsable (Clark et al., 1996), se han reducido las poblaciones y la distribución de varias de estas especies (Nowell \& 
Jackson, 1996; Treves \& Karanth, 2003; Novaro et al., 2004).

Por otro lado, los carnívoros pueden aportar beneficios económicos, emocionales, intelectuales, culturales y espirituales esenciales para el desarrollo y bienestar de los seres humanos (Kellert et al., 1996). Particularmente en las zonas rurales de América del Sur, los pobladores suelen usar a los carnívoros silvestres con fines medicinales, artesanales, culturales y/o religiosos (Iriarte, 1998; Villalba \& Bernal, 1999; García-Perea, 2002; Cossíos \& Madrid, 2002; Villalba et al., 2004; Cossíos et al., 2007).

En el área de estudio, las principales especies de carnívoros, son la comadreja (Mustela frenata Lichtenstein 1831), el gato de pajonal (Lynchailurus colocolo Molina 1782), el puma (Puma concolor Linnaeus 1771), el zorro andino (Dusycion culpaeus Molina 1782) y el zorrillo (Conepatus chinga Molina 1782). En el región andina del Perú, éstos se distribuyen principalmente en los valles interandinos, estepas y zonas montañosas de la puna hasta los 4200 msnm, pudiendo alcanzar incluso mayores altitudes (Pearson, 1957; Grimwood, 1969; Cossíos et al., 2007).

En esta zona, la gran mayoría de los pobladores tienen escaso acceso a la educación y se dedican principalmente al desarrollo de actividades agropecuarias, por lo que los conflictos con los carnívoros altoandinos suelen ser muy comunes. Es por esta razón, que entender el rol de los carnívoros en el ecosistema no es suficiente para resolver los conflictos con el ser humano (Conforti \& Azevedo, 2002). El principal desafío consiste en la integración y participación activa de los pobladores que viven en simpatría con estas especies (Rasker \& Hackman, 1996).

El presente trabajo tiene como finalidad contribuir y complementar los conocimientos y comprensión de los conflictos, actitudes y usos tradicionales que poseen los pobladores rurales sobre los carnívoros altoandinos con el propósito de formular futuras estrategias que aseguren la participación de las comunidades rurales en la conservación de estas especies.

\section{Métodos}

El área de estudio comprendió tres centros poblados en el distrito de Anco, dentro del departamento de Ayacucho, ubicado en los Andes del centro - sur del Perú. En Agosto de 2006, se realizaron 75 entrevistas semi-estructuradas (orales- en castellano) a los pobladores rurales únicamente de sexo masculino (25-70 años) debido a la facilidad que éstos poseen para comunicarse en castellano y porque sus actividades son realizadas principalmente en el campo.
El tamaño óptimo de entrevistados fue calculado a través de una variación en la fórmula de la semiamplitud del intervalo de confianza y la fórmula de la precisión requerida para estimar la media de la población. Ambas fórmulas son comúnmente utilizadas para calcular el tamaño de una muestra aleatoria (Paine \& Kiser, 2003).

Se elaboró un cuestionario que sirvió de guía durante las entrevistas. Para proporcionarle confiabilidad y validez al cuestionario elaborado, éste se sometió a una revisión por parte de cinco especialistas en el tema.

Las entrevistas se ejecutaron en los propios hogares de los pobladores y se condujeron de forma personal, confidencial, interactiva y de fácil comprensión, preguntándoles sobre los conflictos, actitudes y posibles usos dados a los carnívoros altoandinos. Asimismo, se utilizó una cartilla con fotos de los carnívoros en estudio para facilitar su reconocimiento.

La Tabla 1 presenta una breve descripción de las características de los centros poblados visitados.

Tabla 1: Ubicación del área de estudio.

\begin{tabular}{|c|c|c|c|}
\hline Zona de Vida & Centro poblado & Coordenadas & $\begin{array}{l}\text { Altitud } \\
\text { (msnm) }\end{array}$ \\
\hline $\begin{array}{l}\text { Zona de } \\
\text { Transición }\end{array}$ & Chiquintirca & $\begin{array}{l}\text { S 133'37.5’, } \\
\text { W 7342’24.5', }\end{array}$ & 3230 \\
\hline Pajonal & Auquiraccay & $\begin{array}{l}\text { S } 13^{\circ} 05^{\prime} 50.3^{\prime \prime}, \\
\text { W } 73^{\circ} 45^{\prime} 31.0^{\prime \prime}\end{array}$ & 3878 \\
\hline Matorral & Sacharaccay & $\begin{array}{l}\text { S } 13^{\circ} 04^{\prime} 46.2^{\prime \prime}, \\
\text { W } 73^{\circ} 50^{\prime} 59.0^{\prime \prime}\end{array}$ & 3050 \\
\hline
\end{tabular}

\section{Resultados}

Conflictos entre los pobladores rurales y los carnívoros altoandinos:

El 100\% de los entrevistados afirmó que existe un gran conflicto en la convivencia con los carnívoros altoandinos. Este se debe únicamente a la incidencia de pérdidas económicas producidas por la depredación de los animales domésticos y/o pérdidas en la producción de los cultivos agrícolas. El 78.6\% aseguró que la población de carnívoros ha disminuido considerablemente en los últimos años y también las pérdidas económicas que éstos producen.

Según la información obtenida, la comadreja, el gato de pajonal, el puma y el zorro andino serían los cuatro carnívoros altoandinos responsables de la depredación de animales domésticos, mientras que el zorrillo sería el principal causante de las pérdidas en la producción de los cultivos agrícolas.

El puma y el zorro andino son considerados los más perjudiciales debido al número de animales que depredan en un mismo ataque, el tipo de animales que depredan y porque acostumbran a matar más animales de los que suelen consumir. Por otro lado, la comadreja y el gato de pajonal son también considerados perjudiciales pero en menor proporción, 
Ecol. apl. Vol. $7 \mathrm{~N}^{\text {os }} 1$ y 2, pp. 43-48

porque el tipo de animales que depredan no causan pérdidas muy significativas en la economía y subsistencia de los pobladores.

La gran mayoría de los pobladores expresaron que la comadreja es comúnmente conocida por depredar cuyes y gallinas; el gato de pajonal podría depredar gallinas y raras veces ovinos jóvenes; el puma puede depredar animales mayores como burros, caballos y becerros, aunque también ovinos y caprinos adultos, mientras que el zorro andino es conocido por depredar ovinos, caprinos y gallinas. Asimismo, comentaron que los ataques de la comadreja y el zorro son frecuentes durante todo el año, mientras que los ataques de gato de pajonal y puma son más frecuentes en épocas de lluvia (Octubre-Marzo).

El 92\% de los pobladores del distrito de Anco reconoció que no posee ningún registro de posesión, natalidad y mortandad de sus animales domésticos. Asimismo, el $88 \%$ aseguró que las enfermedades sanitarias y la sequía producen mayores pérdidas en el ganado y cultivos en comparación con las producidas por los carnívoros altoandinos.

Relaciones entre los pobladores rurales y los carnívoros altoandinos:

Las principales reacciones de los pobladores luego de una pérdida económica provocada por los carnívoros altoandinos son: mejorar la protección de sus animales y/o cultivos (66.7\%), tratar de matar al carnívoro responsable (26.7\%) o no realizar ninguna acción en particular (6.6\%).

En caso de encuentro o avistamiento de algún carnívoro altoandino, el $33.3 \%$ de los pobladores declaró que intenta perseguirlo y cazarlo sin importar la especie y sin ningún motivo en particular, mientras que al $66.7 \%$ le sorprende su presencia pero no intenta cazarlo.

El 52\% (39 pobladores, específicamente 9 en Chiquintirca, 14 en Auquiraccay y 16 en Sacharaccay) reconoció haber cazado algún carnívoro altoandino en el periodo de Agosto 2005 - 2006 debido a las pérdidas económicas que éstos suelen producir. Ninguno de los entrevistados confirmó tener alguna creencia que los obligue necesariamente a cazar carnívoros altoandinos, ni reportó casos de ataques de carnívoros hacia los seres humanos.

La Tabla 2 presenta los métodos más utilizados por los pobladores rurales para dar caza a los carnívoros altoandinos.

Por otro lado, el $44 \%$ de los pobladores tiene una actitud negativa hacia los carnívoros altoandinos (sin importar la especie), debido a su comportamiento depredador y a las pérdidas económicas que suelen producir, mientras que el $56 \%$ posee una actitud positiva basada en la atracción y apreciación de estas especies debido a su inteligencia, fuerza, poder, valentía y belleza.

Usos de los carnívoros altoandinos:
Tabla 2: Métodos utilizados para cazar a los carnívoros altoandinos.

\begin{tabular}{|c|c|c|c|c|c|}
\hline $\begin{array}{l}\text { Métodos } \\
\text { de caza }\end{array}$ & 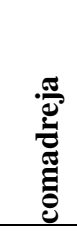 & 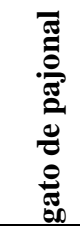 & 䄈 & ํํㄹ & 을 \\
\hline Bosta & & & & & $\mathrm{x}$ \\
\hline Escopeta & & $\mathrm{x}$ & $\mathrm{x}$ & $\mathrm{x}$ & \\
\hline Honda & $\mathrm{X}$ & & & & \\
\hline Humo & & & & $\mathrm{x}$ & \\
\hline Palo & & & & & $\mathrm{x}$ \\
\hline Perro & & $\mathrm{x}$ & & $\mathrm{x}$ & \\
\hline Piedra & & & & & $\mathrm{x}$ \\
\hline Trampa & $\mathrm{x}$ & & & & $\mathrm{x}$ \\
\hline Veneno & & $\mathrm{x}$ & $\mathrm{x}$ & $\mathrm{x}$ & \\
\hline
\end{tabular}

El 100\% de los entrevistados afirmó que la intensidad y valoración del uso de los carnívoros altoandinos ha disminuido en los últimos años. A pesar de esto, su grasa es utilizada con fines medicinales para tratar problemas de reumatismo, artritis y para aliviar dolores musculares y de huesos. Según los pobladores, las grasas más utilizadas para aliviar dichos males son las del puma (76\%) y zorrillo (24\%).

Por otro lado, el $10.4 \%$ de los entrevistados declaró que la sangre y el caldo de zorrillo (4\%) sirven para tratar la tuberculosis, epilepsia y problemas al corazón, hígado y riñones.

Todos los entrevistados declararon que las pieles de los carnívoros altoandinos poseen una importancia cultural y artesanal, ya que suelen exhibirse como adornos en las casas y negocios. Asimismo, no reportan el uso de éstas durante ceremonias religiosas, pero si durante fiestas tradicionales como las patronales y las de carnaval. Adicionalmente, argumentaron que éstas obtendrían un valor comercial, si las vendieran a comerciantes, turistas o investigadores.

El 10.7\% comentó que con la piel de la comadreja se fabrican monederos para guardar dinero y hojas de coca, con la creencia de que estos recursos no falten en la vida diaria. Las garras y colmillos del puma son usados como amuletos de protección contra la brujería, mal de ojo, susto y otras "fuerzas negativas" según el $10.7 \%$, mientras que el $60 \%$ aseguró que el penacho negro de la cola extraída de un zorro vivo sirve como amuleto de buena suerte para la "atracción del amor" y la "prosperidad en los negocios".

Aunque la carne de los carnívoros altoandinos no es una fuente importante de proteína para los pobladores rurales, en raras ocasiones, puede ser consumida como chicharrón frito. Las más 
aprovechadas debido a su "rendimiento y exquisito sabor" son las del puma (57.3\%) y zorrillo (37.3\%).

Por otro lado, el $28 \%$ indicó que existe una práctica ocasional de capturar los cachorros de zorro andino para ser criados como mascotas o ser vendidos ilegalmente en las ciudades por un mínimo precio de 50 soles.

\section{Discusión}

Para los pobladores rurales, la agricultura y ganadería representan grandes fuentes de alimentación y economía, siendo las únicas alternativas de ahorro y capitalización. El ganado doméstico les permite obtener ingresos con poco costo y mano de obra, genera la fuerza de labranza de la tierra, es productor de guano, combustible, da un estatus social y es fuente de distracción en las fiestas patronales.

Sin embargo, la crianza y explotación de los animales domésticos está sujeta a la costumbre, siendo de carácter extensivo y rudimentario. La gran mayoría de los pobladores (92\%) no posee registros de posesión, natalidad o mortandad de sus animales, ni planes de manejo definidos o métodos de protección y prevención contra posibles ataques de depredadores. El ganado al andar libremente por las pasturas, no presenta ningún impedimento para que pueda ser depredado por los carnívoros altoandinos. A pesar de esto, los carnívoros no son el único problema que tiene el ganado, ya que el $88 \%$ reconoció que las enfermedades sanitarias y la sequía producen mayores pérdidas económicas en comparación con las producidas por los carnívoros.

Por otro lado, el ganado doméstico genera efectos negativos sobre el alimento y la cobertura de los carnívoros, reduciendo la densidad e incrementando la vulnerabilidad de las presas silvestres (Pía et al., 2003). Los pobladores suelen restar terreno para sembrar sus campos o extienden el área de pastoreo destinado al ganado, provocando indirectamente la reducción de presas silvestres e impulsando a los carnívoros hacia la depredación del ganado debido a la escasez de alimento.

Según la información obtenida, la comadreja, el gato de pajonal, el puma y el zorro andino presentan comportamientos depredadores hacia los animales domésticos. Butler (2000), señala que si las presas naturales tienen una excelente plenitud dentro de sus hábitats, los carnívoros cazaran éstas con mayor preferencia, lo cual refleja algún tipo de desequilibrio en el ecosistema andino. Esto puede generar consecuencias negativas, ya que la puna por su elevado número de endemismos y su fragilidad, es considerada uno de los ambientes con prioridad de conservación a nivel internacional (Biodiversity Support Program et al., 1995).

En la actualidad, no se conoce el estado ni la distribución de las poblaciones de los carnívoros altoandinos en estudio, sin embargo el $78.6 \%$ de los pobladores aseguró que su avistamiento y las pérdidas económicas que éstos producen también han disminuido considerablemente en los últimos años. A pesar de que mejorar la protección de sus animales y/o cultivos es la principal acción preventiva luego de una pérdida económica provocada por estas especies, más de la mitad de pobladores (52\%) los han cazado con la finalidad de resolver el conflicto de manera temporal.

Conocer la actitud del ser humano hacia una especie de carnívoro determinada es de gran importancia (Cossíos, 2004), ya que los carnívoros funcionan particularmente como un poderoso barómetro para medir las actitudes de los pobladores hacia su medio natural (Kellert et al., 1996).

El $44 \%$ de los pobladores tiene una actitud negativa hacia los carnívoros altoandinos debido a su comportamiento depredador y las pérdidas económicas que producen. La misma percepción y actitud negativa debido al comportamiento depredador ha sido reportada para el jaguar (Hoogesteijn, 1993; Conforti \& Azevedo, 2002), puma (Conforti \& Azevedo, 2002; Mazzolli et al., 2002; SilvaRodríguez et al., 2007; Lucherini \& Merino, 2008), zorro andino (Novaro et al., 2004; Lucherini \& Merino, 2008), zorro de Sechura (Cossíos, 2004), gato de pajonal (Cossíos et al., 2007; Lucherini \& Merino, 2008), gato guigna (Silva-Rodríguez et al., 2007), entre otros. Esto es comprensible, debido a que las muertes de los animales domésticos ocurren principalmente dentro de las propiedades de los pobladores. De esta manera, se genera por obvias razones económicas y psicológicas, un gran resentimiento de parte de los pobladores hacia los depredadores (Mishra, 1997).

A pesar de esto, existe un mayor porcentaje de pobladores (56\%) que posee una actitud positiva hacia los carnívoros altoandinos. Esta es generada debido al afecto a la naturaleza, entendimiento del rol ecológico, creencias morales, simbolismos asociados a estas especies como la inteligencia, fuerza, resistencia y el valor o simplemente por tolerancia (Clark et al., 1996; Kellert et al., 1996; Conforti \& Azevedo, 2002; Lucherini \& Merino, 2008).

No obstante los carnívoros altoandinos suelen ser cazados principalmente por las pérdidas económicas que pueden producir; la grasa, sangre, carne, piel y derivados pueden ser usados por los pobladores rurales con otros fines. La grasa de los carnívoros es comúnmente utilizada para aliviar el reumatismo, dolor de huesos, golpes y torceduras (Cossíos \& Madrid, 2002; Barbarán, 2004).

Los pobladores rurales del distrito de Anco utilizan las pieles de los carnívoros en estudio como adornos en los hogares y negocios, como amuletos de buena suerte y también durante fiestas tradicionales. Asimismo, se reporta el uso de pieles y derivados de felinos durante ceremonias religiosas y danzas 
tradicionales en otras ciudades del Perú y otros países de América del Sur (Iriarte, 1998; Villalba \& Bernal, 1999; Cossíos \& Madrid, 2002; García-Perea, 2002; Villalba et al., 2004; Cossíos et al., 2007). Igualmente, las garras y colmillos del puma son usados como amuletos contra la brujería y para prevenir daños y maldiciones, de la misma forma que reporta Barbarán (2004) en los pobladores de la puna del noroeste Argentino y sur de Bolivia.

Cossíos (2004), señala que existe una práctica ocasional de recoger cachorros del zorro de Sechura (Pseudalopex sechurae) para ser criados como mascotas o ser vendidos ilegalmente en las zonas norte y centro del Perú. En el área de estudio, esta acción es también realizada con las crías del zorro andino. Asimismo, el uso de la cola de ambos cánidos tiene la misma connotación, descrita por Valdizán \& Maldonado (1922).

Por todo lo anteriormente descrito, el reto de la conservación de los carnívoros altoandinos rebasa los aspectos biológicos e involucra también aspectos culturales, sociales y económicos. Es por esta razón, que es necesario contar con otras disciplinas y perspectivas para comprender y minimizar los conflictos que afectan la convivencia entre los seres humanos y estas especies (Clark \& Primm, 1996).

El ser humano cumple un rol muy importante dentro de los ecosistemas y así como es parte del conflicto también puede ser parte de la solución. Por lo tanto, las propuestas que no involucren los intereses de las comunidades rurales serán insignificantes, ya que sólo la gente lugareña tiene la posibilidad de subsanar los conflictos a través de cambios en su comportamiento o el de los carnívoros (Sillero, 2000).

\section{Conclusiones}

La falta de investigación, la fragmentación del hábitat por la expansión de las fronteras agrícolas, la caza y las actitudes negativas amenazan el estado de las poblaciones de los carnívoros, poniendo en riesgo el equilibrio de los ecosistemas andinos.

Los carnívoros altoandinos son considerados perjudiciales porque interfieren en las actividades agropecuarias de los seres humanos. El principal conflicto entre los pobladores se debe a las pérdidas económicas producidas por la depredación de animales domésticos y/o pérdidas en la producción de los cultivos agrícolas. Debido a este conflicto, el $44 \%$ presenta una actitud negativa y el 52\% los ha cazado con la finalidad de resolver el conflicto de manera temporal. A pesar de ello, el 56\% posee una actitud positiva basada en percepciones de atracción y apreciación por la inteligencia, fuerza, poder, valentía y belleza de estas especies.

Esto demuestra claramente la necesidad de tomar acciones integradoras que generen un cambio perceptual y actitudinal de las comunidades rurales frente a los carnívoros altoandinos.
Es por esta razón, que los pobladores deben ser vistos como aliados para la conservación y no lo contrario, ya que su participación y cooperación a largo plazo permitirá la ejecución de planes de manejo y programas de educación ambiental, con el propósito de generar estrategias que permitan el bienestar de los pobladores y la conservación de los carnívoros altoandinos.

Aunque la intensidad y valoración del uso de los carnívoros altoandinos ha disminuido en los últimos años, éstos aún son aprovechados con fines medicinales a través de la grasa; alimenticios a través de la carne y culturales y/o artesanales a través de la piel. En este sentido, se debe combinar el uso tradicional con la práctica de la conservación, para integrar a los pobladores rurales con el bienestar de los ecosistemas andinos y revalorizar el rol de los carnívoros altoandinos en su cultura.

\section{Recomendaciones}

Debido a la escasa información sobre los carnívoros altoandinos, se recomienda continuar desarrollando estudios que comprometan la conservación de estas especies, como son:

- Distribución ecológica y geográfica.

- Abundancia y densidad de las poblaciones.

- Cuantificación de pérdidas económicas por depredación de animales domésticos.

- Métodos de control indirectos que minimicen la depredación sobre animales domésticos.

\section{Agradecimientos}

A la Universidad Nacional San Cristóbal de Huamanga y la Municipalidad del distrito de Anco, por la facilidades que brindaron durante la ejecución de este proyecto. A todos los pobladores rurales del distrito de Anco que aceptaron ser entrevistados y demostraron una gran disposición e interés. A Aldo López por su constante apoyo y compañía. Asimismo, a todos los profesionales y expertos que brindaron sus observaciones y contribuciones, con especial mención a Consuelo Arellano, Daniel Cossíos y Samuel Astete.

\section{Literatura citada}

Barbarán F.R. 2004. Usos mágicos, medicinales y rituales de la fauna en la Puna del Noroeste Argentino y Sur de Bolivia. Contribuciones al Manejo de Vida Silvestre en Latinoamérica. 1(1): 01 - 26.

Biodiversity Support Program, Conservation International, The Nature Conservancy, Wildlife Conservation Society, World Resources Institute \& World Wildlife Fund. 1995. A regional analysis of geographic priorities for biodiversity conservation in Latin America and the Caribbean. Biodiversity Support Program. Washington, D.C., USA.

Butler J.R.A. 2000. The economic costs of wildlife predation on livestock in Gokwe communal land, Zimbabwe. African Journal of Ecology. 38: 23 - 30. 
Clark T.W., Curlee A.P. \& Reading R.P. 1996. Crafting effective solutions to the large carnivore conservation problem. Conservation Biology. 10: 940 - 948.

Clark T.W. \& Primm S.A. 1996. Making sense of the policy process for carnivore conservation. Conservation Biology. 10: 1036 - 1045.

Conforti V.A. \& de Azevedo F.C.C. 2002. Local perceptions of jaguars (Panthera onca) and pumas (Puma concolor) in the Iguaçu National Park Area, South Brazil. Biological Conservation. 111: 215 - 221.

Cossíos E.D. 2004. Relaciones entre el zorro de sechura, Pseudalopex sechurae y el hombre en el Perú. Ecología Aplicada. 3: 134 - 137.

Cossíos E.D. \& Madrid A. 2002. Andean mountain cat (Oreailurus jacobita) and other andean carnivores status survey in Ayacucho, Arequipa, Puno and Tacna departments. Perú. Report to Cat Action Treasury. 17 pp.

Cossíos D.E., Madrid A., Condori J.L. \& Fajardo U. 2007. Update on the distribution of the Andean cat (Oreailurus jacobita) and the pampas cat (Lynchailurus colocolo) in Peru. Endangered Species Research. 3: 313 - 320.

García-Perea R. 2002. Andean mountain cat, Oreailurus jacobita: morphological description and comparison with other felines from the altiplano. Journal of Mammalogy. 83 (1): 110 - 124.

Grimwood I.R. 1969. Notes on the distribution and status of some Peruvian mammals. In Special Publication $\mathrm{N}^{\mathrm{o}} 21$. New York Zoological Society, New York.

Hoogesteijn R. 1993. Manual sobre problemas de depredación causados por jaguares y pumas en hatos ganaderos. In Wildlife Conservation Society: 01 - 38.

Iriarte A.W. 1998. Distribution and status of the Andean mountain cat in Chile. Report to Cat Action Treasury. Disponible http://www.felidae.org/LIBRARY/irirptsp.html.

Kellert S.R., Black M., Rush C.R. \& Bath A.J. 1996. Human culture and large carnivore conservation in North America. Conservation Biology 10: 977 - 990.

Kruuk H. 1976. Carnivores and conservation. En: Proceedings of a symposium on endangered wildlife of South Africa. pp 1-13. The Endangered Wildlife Trust and University of Pretoria, Pretoria.

Linnell J.D., Swenson J.E. \& Anderson R. 2001. Predators and people: conservation of large carnivores in possible at high human densities if management policy is favorable. Animal Conservation: 345 - 349.

Lucherini M. \& Merino M.J. 2008. Perceptions of HumanCarnivore conflicts in the High Andes of Argentina. Mountain Research and Development. 28 (1): 81-85.

Mazzolli M., Graipel M.E. \& Dunstone N. 2002. Mountain lion depredation in southern Brazil. Biological Conservation. 105: 43 - 51.

Mishra C. 1997. Livestock depredation by large carnivores in the Indian trans - Himalaya: conflict perceptions and conservation prospects. Environmental Conservation. 24 (4): $338-343$.

Novaro A.J., Funes M.C. \& Jiménez J.E. 2004. Patagonia foxes. Selection of introduced prey and conservation of culpeo and chilla foxes in Patagonia. Biology and Conservation of Wild Canids: 243-254.

Nowell K. \& Jackson P. 1996. Status and Conservation Action Plan: Wild Cats. Gland, Switzerland: UICN/ SSC Cat Specialist Group. 382 pp.

Oil M.K., Taylor I.R. \& Rogers M.E. 1994. Snow leopard (Panthera uncia) predation of livestock: an assessment of local perceptions in the Annapurna conservation area, Nepal. Biological Conservation. 68: 63 - 68.

Ojasti L. 1984. Hunting and conservation of mammals in Latin America. Acta Zool. Fennica. 172: 177 - 181.

Paine D.P. \& Kiser J.D. 2003. Aerial Photography and Image Interpretation for Resource Management. Segunda Edición. New Jersey. 648 pp.

Pearson O.P. 1957. Additions to the mammalian fauna of Peru and notes on some other Peruvian mammals. Breviora. Museum of Comparative Zoology $\mathrm{N}^{\circ} 73$.

Pía M.V., López M.S. \& Novaro A.J. 2003. Efectos del ganado sobre la ecología trófica del zorro culpeo (Pseudalopex culpaeus smithersi) (Carnivora: Canidae) endémico del centro de Argentina. Revista Chilena de Historia Natural. 76: 313 - 321.

Rasker R. \& Hackman A. 1996. Economic development and the conservation of large carnivores. Conservation Biology. 10: 991 - 1002.

Sillero C. 2000. Editorial sobre resolución de conflictos entre los grandes carnívoros y el hombre. Mastozoología Neotropical. 7 (2): 69 - 72.

Silva-Rodríguez E.A., Ortega-Solís G.R. \& Jiménez J.E. 2007. Human attitudes toward wild felids in a humandominated landscape of Southern Chile. Cat News. 46: 19-21.

The UICN / SSC Canid Specialist Group. 1990. Predation and predator control from foxes, wolves, jackals and dogs.: $1-12$.

Treves A. \& Karanth K.U. 2003. Human - carnivore conflict and perspectives on carnivore management worldwide. Conservation Biology. 17: 1491 - 1499.

Valdizán H. \& Maldonado A. 1922. La medicina popular peruana. Torres Aguirre, Lima.

Villalba L. \& Bernal N. 1999. Distribution and status of the Andean mountain cat in Bolivia. Report to Cat Action Treasury. 10 pp.

Villalba L., Lucherini M., Walker S., Cossíos D., Iriarte A., Sanderson J., Gallardo G., Alfaro F., Napolitano C. \& Sillero-Zubiri C. 2004. The Andean Cat: a conservation action plan. Andean Cat Alliance, La Paz. 83 pp.

Woodroofe R. \& Ginsberg J.R. 1998. Edge effects and the extinction of populations inside protected areas. Science. 280: 2126 - 2128.

\footnotetext{
${ }^{1}$ Universidad Nacional Agraria La Molina. (UNALM), Perú. irisviruta@hotmail.com

${ }^{2}$ Departamento Académico de Biología. Facultad de Ciencias. Universidad Nacional Agraria La Molina. Apartado postal 12-056. Lima 12 - Perú. mwilliams@lamolina.edu.pe

${ }^{3}$ Departamento Académico de Manejo Forestal. Facultad de Ciencias Forestales. Universidad Nacional Agraria

La Molina. Apartado postal 12-056. Lima 12 - Perú. cdc@lamolina.edu.pe
} 